\title{
La contribución internacional de América Latina y el Caribe a los estudios métricos de la información. El caso de la revista Scientometrics
}

The international contribution of Latin America and the Caribbean to metric information studies. The case of Scientometrics journal

Sandra Edith Miguel

Instituto de Investigaciones en Humanidades y Ciencias Sociales -IdIHCS (UNLP-CONICET), Universidad Nacional de la Plata, Argentina

smiguel@fahce.unlp.edu.ar

(iD http://orcid.org/0000-0001-9384-6838

\section{Gustavo Liberatore}

Universidad Nacional de Mar del Plata. Departamento de Ciencia de la Información, Argentina gliberat@mdp.edu.ar

iD http://orcid.org/0000-0002-5841-8739

\section{Resumen:}

El objetivo de esta investigación es caracterizar la contribución científica en estudios métricos de la información (EMI) de los países de América Latina y el Caribe en la revista Scientometrics, desde 1978 hasta la actualidad, observando la participación de cada país, el perfil temático y naturaleza de las investigaciones, y las fuentes que constituyen la base intelectual de referencia de dicha producción. Los resultados muestran que 11 países de la región han realizado al menos una contribución a la revista, con predominio de trabajos procedentes de Brasil, México, Cuba, Chile, Colombia y Argentina. La tendencia de crecimiento es exponencial a partir de la década de 2000. Desde el perfil temático, predominan los estudios de naturaleza aplicada sobre la producción de dominios disciplinares e institucionales. Entre las fuentes que constituyen la base intelectual se encuentra la propia revista Scientometrics y otras revistas nucleares del campo a nivel internacional, y otras revistas de otras disciplinas. Hay muy poca presencia de revistas regionales. Se concluye que, si bien la presencia de autores de la región en la revista es escasa, se evidencia el interés de la comunidad latinoamericana dedicada a los EMI en dialogar con la comunidad internacional especializada en este campo de estudios.

Palabras ClaVe: Bibliometría, Producción científica, Scientometrics, América Latina y el Caribe.

\section{ABStraCt:}

The main objective of the present research is to characterise the scientific contributions regarding metric information studies of Latin American and the Caribbean to Scientometrics journal, since 1978 to the present and from the perspective of each country's participation, the thematic profile and nature of the research and the journals that constitute the production's intellectual foundation. Results show that 11 countries from the region have contributed at least once to the journal, with a predominance of Brazil, Mexico, Cuba, Chile, Colombia and Argentina. Since the 2000s, the growth trend is exponential. Concerning the thematic profile, applied studies about the production of disciplinary and institutional domains prevail. Among the sources that constitute the intellectual foundation, Scientometrics journal itself and other international core journals of the field, as well as journals from other disciplines, are found. There's little presence of regional journals. In conclusion, although there's a scarce presence of regional authors in the journal, the Latin-American community devoted to MIS shows clear interest in generating a discussion with the international community specialised in this field of study.

KEYwORDs: Bibliometric analysis, Scientific production, Scientometrics, Latin America and the Caribbean. 


\section{INTRODUCCIÓN}

El campo de los estudios métricos de la información (EMI) constituye, en la actualidad, un dominio de conocimiento dedicado al estudio, análisis y representación de los distintos fenómenos vinculados con la producción, circulación y consumo de información de las actividades científicas, tecnológicas y de innovación. Comprende desde los aportes teóricos y metodológicos más tradicionales de la bibliometría, cienciometría e informetría hasta las más recientes miradas que giran en torno a la webmetría y las métricas alternativas; como también las contribuciones centradas en la discusión y debate de los alcances de aplicación y utilidad de los indicadores, métodos y productos en diferentes contextos.

La literatura internacional recoge varios estudios que analizan el desarrollo de este campo de estudios en el mundo. Schoepflin y Glänzel (2001) señalan que, desde sus comienzos, los EMI se caracterizaron por aportes procedentes de diversas disciplinas como las matemáticas, la sociología, la psicología y la ciencia bibliotecológica. Desde la década de 1960 hasta finales de los ochenta se fueron consolidando como una disciplina científica en sí misma, siendo la aparición de la revista Scientometrics, en 1978, un hito en su proceso de institucionalización social representado en la creación de sus propias estructuras de comunicación. Un análisis de las contribuciones a la revista, durante las dos primeras décadas, revela que el campo de los EMI tiene un amplio y heterogéneo perfil de investigación dentro de las ciencias sociales "duras", abarcando varias subdisciplinas, cada una de ellas con sus propias características. Las aportaciones comprenden desde la formulación de teorías y modelos matemáticos hasta estudios sociales de la ciencia y política científica, siendo el tipo de contribuciones predominantes los estudios de caso, análisis empíricos y trabajos metodológicos y de bibliometría aplicada (Schoepflin y Glänzel, 2001). En 2003, Glänzel, distingue tres perfiles en la investigación bibliométrica: por un lado, la bibliometría para bibliométricas, que comprende la investigación básica con un fuerte componente metodológico; por otro, la bibliometría para las disciplinas, donde la investigación está fuertemente orientada al estudio del desarrollo de la ciencia por disciplinas y especialidades, y finalmente, la bibliometría orientada a la política y gestión de la ciencia, en la que se encuentran los estudios de evaluación de la investigación y de las estructuras institucionales, nacionales y regionales de los sistemas científicos.

El carácter heterogéneo e interdisciplinario de los EMI hace que la literatura producida en el campo se encuentre dispersa en publicaciones diversas, especialmente de diferentes campos disciplinarios. Sin embargo, son predominantes los artículos en revistas (Martín-Martín, Orduña-Malea, Ayllón y Delgado López-Cózar, 2016). Asimismo, en un análisis de la literatura en bibliometría indizada en la base de datos Library and Information Science Abstracts (LISA) abarcando el período 1968-2004, Patra, Bhattacharya y Verma (2006) encuentran que el 41,5\% de los artículos fue publicado en la revista Scientometrics. En segundo y tercer lugar -con el 5\% de las contribuciones cada una- aparecen el Journal of the American Society for Information Science and Technology (JASIS\&T) y la Revista española de documentación cientifica (REDC).

Martín-Martín, Orduña-Malea, Ayllón y Delgado López-Cózar (2016) siguen el modelo de Bradford, y utilizando como fuentes Google Scholar Citations, ResearcherID, ResearchGate, Mendeley, \& Twitter, y encuentran 6 revistas nucleares del campo: Scientometrics, Journal of informetrics, JASIS\&T, Research evaluation, Research policy y Cybermetrics. Le sigue otro grupo de publicaciones de bibliotecología y ciencia de la información: Journal of information science, Information processing \& management, Journal of documentation, College research libraries, Library trends, Online information review, REDC, Aslib proceedings y El profesional de la información. Finalmente, aparecen revistas dedicadas a estudios sociales y políticos de la ciencia: Social studies of science, Science and public policy, Minerva, Journal of health services research policy, Technological forecasting and social change, Science technology buman values, Environmental science policy y Current science.

Vinkler (2019) identifica un conjunto de revistas líderes del campo de los EMI utilizando las bases de datos del Web of Science (WoS), y abarcando el período 1975-2017, a partir del análisis de las publicaciones más 
citadas de un grupo de investigadores destacados en el campo. Los resultados muestran que: Scientometrics, Journal of informetrics, JASIS\&T y Research policy son las más influyentes. Le sigue otro grupo de revistas de las ciencias de la información como el Journal of information science, Journal of documentation y Annual review of information science and technology. En tercer lugar, aparecen prestigiosas revistas generalistas como Nature, Science, Proceedings of the National Academy of the United States, y otras de ciencias médicas como el British medical journal y el Journal of the American Medical Association.

Una característica observable en los diferentes grupos de revistas que publican estudios bibliométricos, excepto el caso de las especializadas como Scientometrics, Journal of informetrics y Cybermetrics (discontinuada en 2015), es que en gran medida son revistas generalistas, ya sea del campo de la bibliotecología y ciencia de la información, como multidisciplinares o de otras disciplinas. Esta particularidad hace que identificar la literatura producida en el campo no resulte sencillo. A lo que se agrega, una notable dispersión terminológica que dificulta la recuperación de información en los diferentes sistemas de información que recogen la producción bibliográfica (Martín-Martín, Orduña-Malea, Ayllón y Delgado López-Cózar, 2016).

\section{La producción sobre EMI en los PAíses de AmÉrica LATINA y EL CARibe}

El conocimiento que existe sobre la producción de países de América Latina y el Caribe (ALyC) en el campo de los EMI es escaso, y presenta también dificultades en su localización, tanto por la diversidad terminológica como de las fuentes que la recogen. No obstante, algunos de los últimos estudios encontrados para el caso de Brasil (Urbizagástegui Alvarado, 2016; Urbizagástegui Alvarado y Restrepo Arango, 2017), México (Restrepo Arango y Urbizagástegui Alvarado, 2016), Colombia (Urbizagastegui y Restrepo Arango; 2013) y Argentina (Miguel y Dimitri, 2013) permiten conocer algunos patrones que caracterizan la contribución latinoamericana al campo.

Para períodos similares que abarcan desde las décadas del setenta y ochenta hasta 2012, el volumen de la producción para Brasil asciende a 2.300 documentos, notablemente superior que la de México (429), Colombia (255) y Argentina (218). El mayor crecimiento de la literatura se produce desde fines de la década de 1990 y principios de 2000.

La producción se aboca a estudios en campos disciplinares diversos: bibliotecología, educación, administración, comunicación social, psicología, medicina y salud pública, ciencias biológicas y naturales, lo que podría dar indicios de una bibliometría aplicada al estudio de las disciplinas siguiendo la clasificación de Glänzel (2003). Para el caso de Brasil, que es el país que presenta mayor cantidad de estudios sobre el desarrollo de la investigación bibliométrica, Grácio y Oliveira (2012) confirman esta característica, encontrando una menor presencia de trabajos que aportan al desarrollo del propio campo y los orientados a la política científica.

Si bien existe una gran dispersión de la literatura, los tipos documentales de preferencia son los artículos de revistas publicados en lengua materna, a diferencia de lo encontrado en la literatura internacional indizada en la base de datos LISA, donde el inglés aparece como la lengua dominante (Patra, Bhattacharya y Verma, 2006). Las principales revistas elegidas para publicar son del propio país o de países de la región, especialmente del campo de la Bibliotecología y Ciencia de la Información, aunque también de otros campos disciplinares. La Tabla 1 presenta las principales revistas según los resultados de los estudios encontrados. Es posible observar que Scientometrics aparece como la primera elegida en Argentina, la segunda en Brasil y México, y la cuarta en Colombia. El resto de las revistas donde publican son distintas, aunque comparten la REDC (Argentina y México) Ciência da informação (Brasil y Argentina). Lamentablemente, de otros países latinoamericanos no se han encontrado trabajos que permitan tener un mayor conocimiento de la investigación en EMI en la región. 
Tabla 1. Principales revistas donde publican investigadores latinoamericanos en el campo EMI

\begin{tabular}{|c|c|}
\hline $\begin{array}{l}\text { Brasil 1973-2012 (Urbizagástegui } \\
\text { Alvarado, 2016) }\end{array}$ & $\begin{array}{c}\text { México 1971-2012 (Retrepo Arango y } \\
\text { Urbizagástegui Alvarado, 2016) }\end{array}$ \\
\hline Ciência da informação & Investigación bibliotecológica \\
\hline Scientometrics & Scientometrics \\
\hline Encontros bibli & REDC \\
\hline Perspectivas em ciência da informação & Biblioteca universitaria \\
\hline Transinformação & Interciencia \\
\hline Em questão & Anales de documentación \\
\hline Revista de administração contemporânea & Salud mental \\
\hline DataGramaZero & Perfiles educativos \\
\hline Informação \& sociedade: estudos & Ciencias de la información \\
\hline RAE-Revista de administração de empresas & Gaceta médica de México \\
\hline Informação \& informação & OMNIA \\
\hline Revista brasileira de biblioteconomia e & Salud pública de México \\
\hline Documentação & Comunicación y sociedad \\
\hline \multirow[t]{3}{*}{ PontodeAcesso } & Avance y perspectiva \\
\hline & Journal of information science \\
\hline & Revista de la Academia Mexicana de Ciencias \\
\hline $\begin{array}{l}\text { Colombia 1982-2012 (Urbizagástegui } \\
\text { Alvarado y Restrepo Arango, 2013) }\end{array}$ & Argentina 1984-2012 (Miguel y Dimitri, 2013) \\
\hline Revista interamericana de bibliotecología & Scientometrics \\
\hline Revista latinoamericana de psicología & Información, cultura y sociedad \\
\hline Universitas psychologica & REDC \\
\hline Scientometrics & Científica \\
\hline Investigación bibliotecológica & Ciência da informação \\
\hline Acta biológica colombiana & Revista argentina de cardiología \\
\hline Acta colombiana de psicología & Medicina (Buenos Aires) \\
\hline Avances en psicología latinoamericana & Acta gastroenterológica latinoamericana \\
\hline Ingeniería e investigación & El profesional de la información \\
\hline Revista colombiana de psicología & Revista argentina de ciencias del \\
\hline Innovar & comportamiento \\
\hline Colombia médica & Psiencia \\
\hline Revista de salud pública & $\begin{array}{l}\text { Anuario de investigaciones de la Facultad de } \\
\text { Psicología de la Universidad de Buenos Aires }\end{array}$ \\
\hline \multicolumn{2}{|l|}{ Revista MVZ Córdoba } \\
\hline Universitas scientiarum & \\
\hline
\end{tabular}

Fuente: elaboración propia.

Un estudio sobre las contribuciones realizadas por los países a la revista Scientometrics entre 1978 y 2010 , muestra un crecimiento lineal en su incorporación, siendo los diez países con mayor representación: Estados Unidos, Bélgica, España, China, Holanda, Inglaterra, Hungría, India, Alemania y Francia (Chen, Börner y Fang, 2013). Las contribuciones de los países de América Latina y el Caribe a la revista no han sido aún exploradas.

\section{OBJETIVOS Y PREGUNTAS DE INVESTIGACIÓN}

Dado el escaso conocimiento de la contribución internacional de los países de América Latina y el Caribe al campo de los EMI, y siendo la revista Scientometrics (la más importante y más antigua publicación 
especializada), elegida como el principal espacio de comunicación de las contribuciones latinoamericanas de los EMI en la comunidad internacional, esta investigación se propone como objetivo: caracterizar las contribuciones científicas en el área EMI de los países de América Latina y el Caribe publicadas en la revista Scientometrics, desde 1978 hasta la actualidad, observando la participación de cada país, el perfil temático y naturaleza de las investigaciones, y las fuentes que constituyen la base intelectual de referencia de dicha producción.

Las preguntas que se propone responder son: ¿Qué países de América Latina y el Caribe contribuyen a la producción científica sobre EMI en la comunidad internacional a través de la revista Scientometrics? ¿Cómo ha sido la evolución de la participación de cada país en la revista? ¿Cuál es el perfil temático y la naturaleza de las contribuciones? ¿Qué revistas u otras fuentes constituyen la base intelectual de la producción?

\section{Materiales y Métodos}

Se realizó una búsqueda utilizando la base de datos Scopus de los trabajos publicados en la revista Scientometrics desde 1978 a 2020, filtrando luego el conjunto de los trabajos en los que figurara al menos un país de América Latina y el Caribe en el campo de país de afiliación de los autores (AfflCountry). Se exportaron los registros a una fuente de datos ad-hoc incluyendo todos los campos. Del total de registros recuperados, cerca de un $25 \%$ carecían de palabras clave en la base de datos. Para salvar este problema, se indizó a este conjunto mediante un análisis directo de los documentos publicados en la revista.

A partir del recuento de los documentos recuperados, se analizó el volumen y distribución temporal de la producción, tanto a nivel regional como por país, en base a los datos de afiliación institucional de los autores firmantes de los trabajos y el año de publicación.

Para representar el perfil temático de los artículos se utilizó el método de análisis de co-ocurrencia de términos (co-word analysis) tomando como base las palabras clave de los documentos asignadas por los autores. La matriz resultante fue procesada mediante el análisis de redes sociales (ARS) utilizando la aplicación Ghepi (versión 0.9.2) y aplicando el algoritmo de distribución Force Atlas. Para ajustar mejor la representación se aplicó un proceso de lematización tendiente a reforzar la consolidación de los diferentes grupos semánticos y disminuir el efecto de la gran dispersión existente producto de las diferentes variantes utilizadas en la asignación de los conceptos asociados a las técnicas, métodos e indicadores bibliométricos. Esta particularidad no sólo se deriva de los distintos enfoques existentes en este campo (bibliométrico, informétrico, cienciométrico) sino además porque los EMI poseen la característica de ser transversales a todos los dominios científicos y son aplicados por investigadores provenientes de diferentes disciplinas lo que produce una gran inflación de expresiones diferentes pero equivalentes. Los resultados fueron además interpretados según el modelo de clasificación de Glänzel (2003) para las tipologías de estudios bibliométricos.

En el análisis de la base intelectual de la producción también se utilizó el método de las co-citas (cocitation analysis) de las fuentes incluidas en la bibliografía de los trabajos, utilizando para la representación y visualización de los resultados la aplicación VosViewer (versión 1.6.15), y se aplicó como punto de corte una frecuencia de citación mínima de 20.

\section{Resultados}

Durante el período 1978-2020 se encontraron 340 trabajos publicados en la revista Scientometrics con participación de al menos un autor procedente de América Latina y el Caribe. En total aparecen 11 países de la región, aunque el peso de sus contribuciones es muy dispar. Como era de esperar, Brasil es el país con 
mayor presencia, seguido de México, Cuba, Chile, Colombia, Argentina, Venezuela, y con menos del 1\%: Puerto Rico, Ecuador, Uruguay y Guatemala (Figura 1).

Figura 1. Distribución porcentual del volumen de la producción por países de América Latina y el Caribe en la revista Scientometrics (1978-2020).

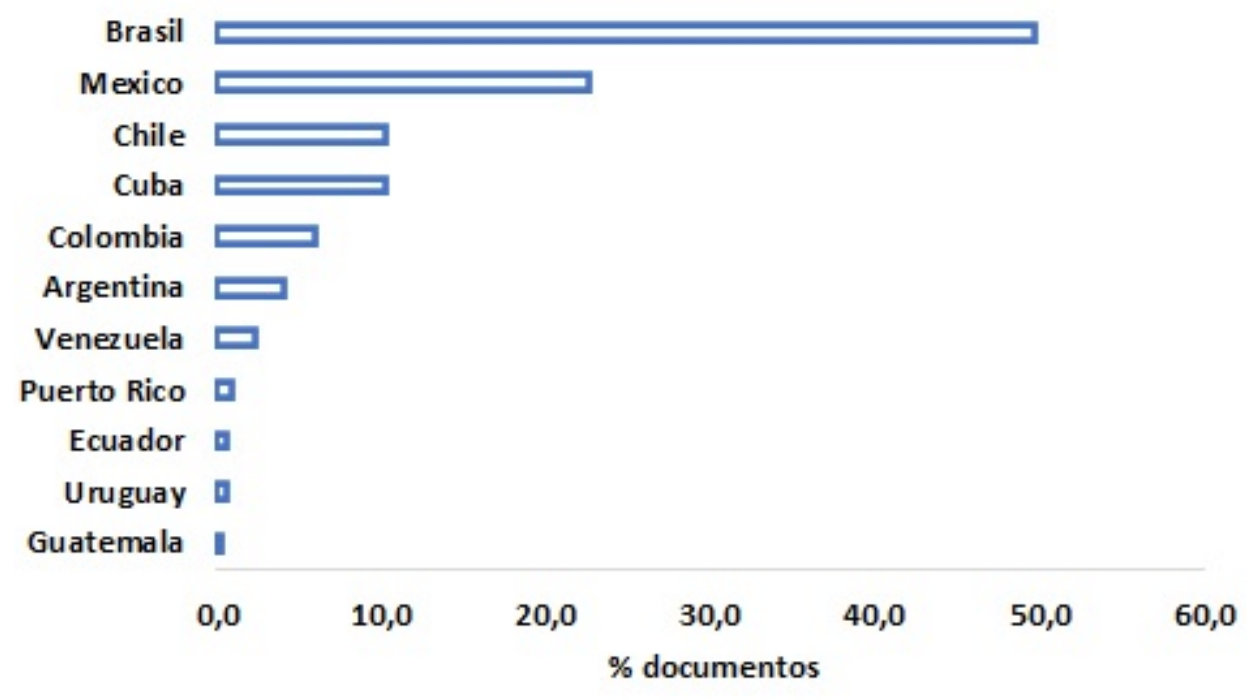

Fuente: elaboración propia.

Se observan dos momentos en la evolución de la producción. El primero, abarca hasta fines de la década del noventa, durante el cual se mantiene un ritmo de crecimiento moderado, siendo recién a partir del quinquenio 2001-2005 cuando aparece una tendencia de crecimiento sostenida, muy a tono con las tendencias internacionales de aplicación de los EMI como instrumento de evaluación y diagnóstico de las actividades científicas (Figura 2).

Figura 2. Evolución de la producción de países de América Latina y el Caribe en la revista Scientometics (1978-2020).

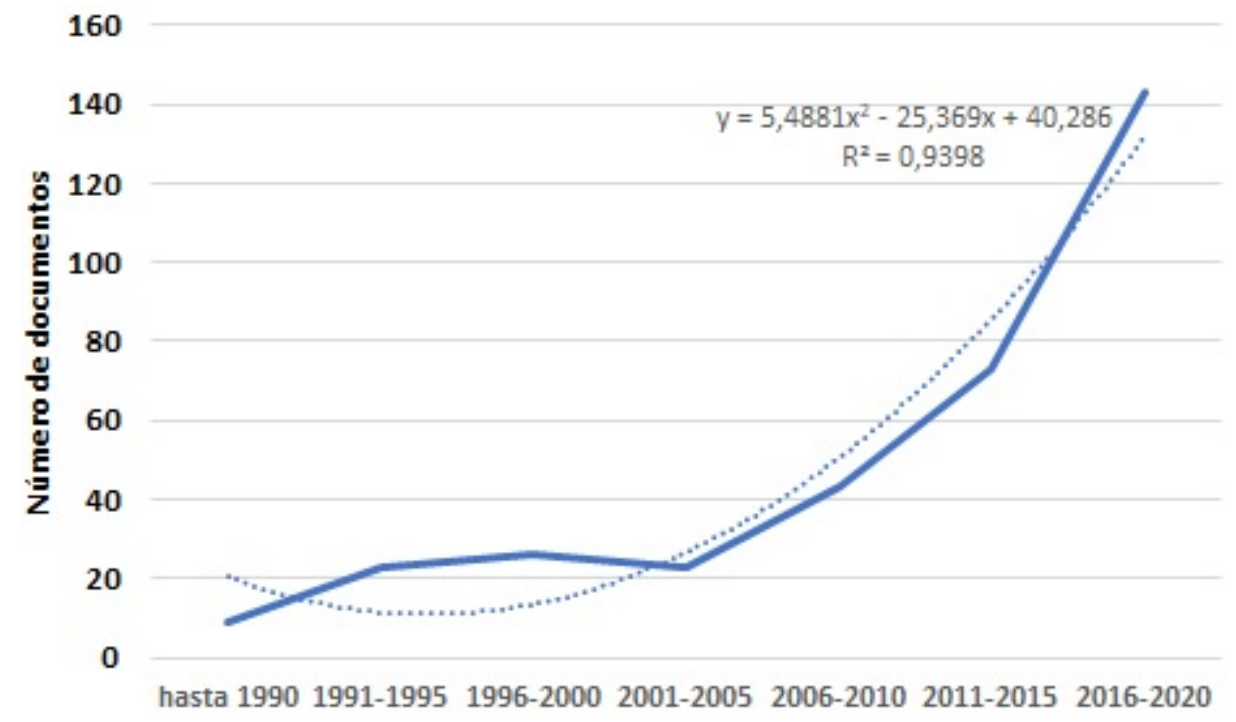

Fuente: elaboración propia. 
No todos los países comenzaron a publicar tempranamente en la revista. Como se observa en la Figura 3, la incorporación de países es gradual y no se mantuvo de manera sostenida, excepto en los casos de Brasil y México, y Cuba que, salvo en el quinquenio 1991-1995, generan una producción constante. Chile aparece en el este último quinquenio y mantiene su presencia a lo largo de las décadas. Colombia realiza su primera aparición entre 1996 y 2000, y luego vuelve a tener contribuciones a partir del quinquenio 2006-2010. Argentina aparece en el quinquenio 1991-1995, y vuelve a tener presencia una década más tarde, similar al comportamiento de Venezuela, con la diferencia que éste último lo hizo un quinquenio después. Los otros países (Puerto Rico, Ecuador, Uruguay y Guatemala) aparecen recién a partir del quinquenio 2011-2015, aunque no es posible interpretar su evolución porque contribuyen con muy pocos trabajos cada uno (entre 1 y 3$)$.

Figura 3. Evolución de la producción por países de América Latina y el Caribe en la revista Scientometrics (1978-2020).

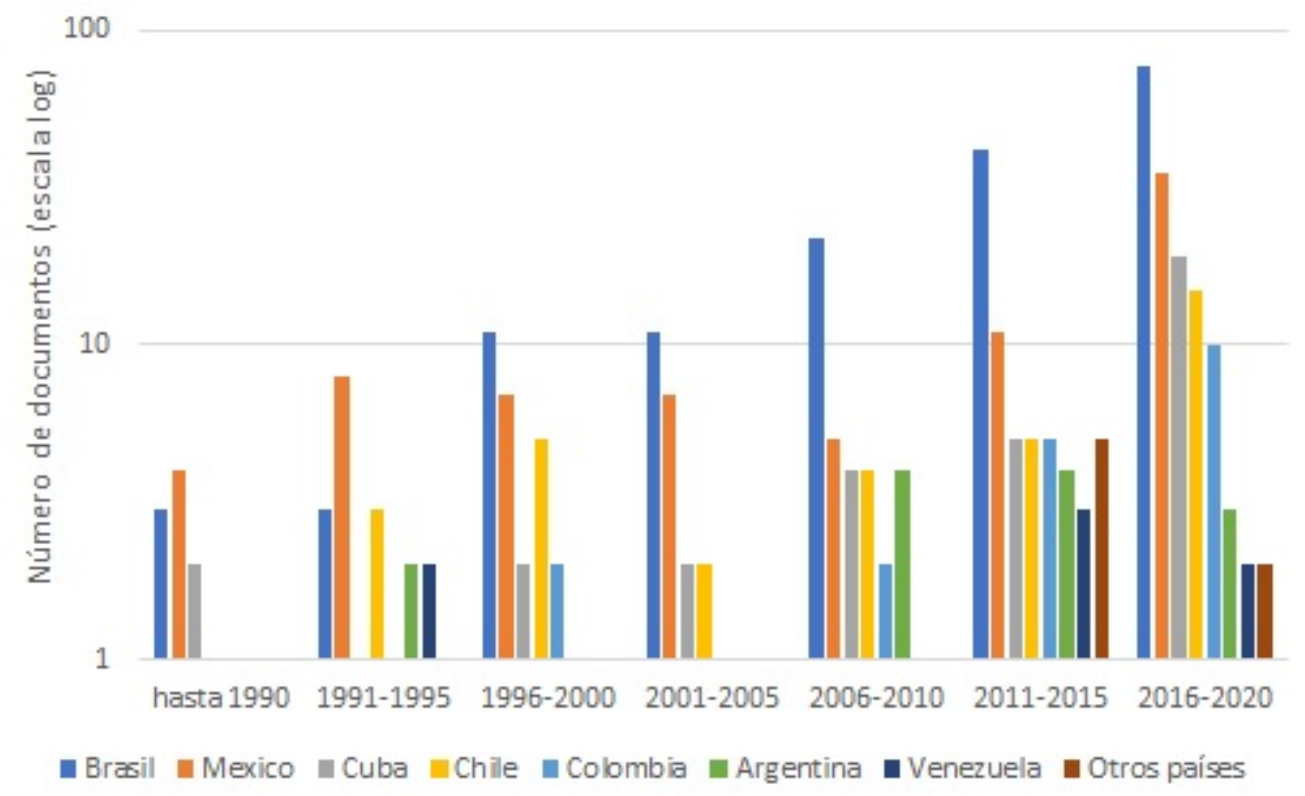

Fuente: elaboración propia.

Para el estudio del perfil temático se recurrió a una representación mediante un grafo generado a partir del análisis de redes sociales (ARS) (Figura 4). El índice primario de palabras clave estuvo constituido por 1091 expresiones lo que arroja un promedio de 3,2 términos por artículo. Luego del proceso de lematización, y para una mejor visualización de las relaciones se aplicó a la red el cálculo de modularidad con el objeto de representar los clusters o agrupamientos subyacentes. El resultado arrojó la delimitación de cuatro subredes principales que representan, en un primer análisis, los principales focos de interés en la aplicación de los EMI dentro de la región. El agrupamiento más importante es el conformado en torno al concepto bibliometric analysis y, al mismo tiempo, el de contornos más difusos dada la amplitud semántica de la expresión. En él se agrupan aquellas investigaciones más amplias en términos de los indicadores observados y de las temáticas y dominios cubiertos. El segundo agrupamiento en importancia es el estructurado sobre la expresión Scientific production, el cual se centra primordialmente en el análisis de las capacidades tanto de sistemas científicos nacionales (se destacan Brasil y México) como de instituciones y dominios temáticos a partir de la medición de la producción científica; en particular aquella indizada en la corriente principal. De allí que los nodos secundarios más destacados sean Impact Factor, International Collaboration e International Visibility representando las variables más estudiadas para medir el desempeño y performance de lo local o regional a nivel internacional, y su relación con las políticas científicas, como lo destacan los nodos Science 
Policy y Policy Maker. En tercer lugar se destaca la subred con centro en el tema Citation analysis enfocada en el análisis de citas, tanto desde la perspectiva de la evaluación científica (Science evaluation) como también de las fuentes (Journals y Latin American Journals). Un aspecto relevante y que confirma que la mayoría de los EMI analizados se basan en las producciones indizadas en la corriente principal es la existencia de nodos con mención de las dos fuentes de datos principales (WoS y $S c o p u s)$, o algunos de sus productos (Science Citation Index y Journal Citation Reports). Este aspecto, por otra parte, se justifica en el hecho de que estas dos bases de datos son las únicas que proveen los datos necesarios para realizar este tipo de análisis. Por último, resulta destacable la aparición de dos países, Cuba y Argentina, como aquellos principalmente "mapeados" desde la perspectiva de las citas. Finalmente, y con menor peso se ubica el agrupamiento Collaboration analysis que revela a una de las temáticas más estudiadas y de mayor especificidad respecto a los demás clusters, y en particular sobre otros indicadores, lo que demuestra que es uno de los aspectos que recibe la mayor atención, sobre todo en la última década.

$\mathrm{Al}$ ajustar el análisis del perfil temático de esta producción a las categorías propuestas por Glänzel (2003) puede observarse que no existe una traducción directa, en gran parte porque la clasificación propuesta por este autor es modélica o abstracta. Aún así, se reconocen dos de los perfiles mencionados como son el de la bibliometría orientada a las disciplinas y aquella enfocada en la política y gestión de la ciencia. Claramente, estas dos categorías atraviesan la totalidad de los artículos estudiados, demostrando la potencialidad de los EMI para el análisis y diagnóstico de las múltiples dimensiones de la actividad científica, en lo que podríamos englobar como investigaciones de "bibliometría aplicada". La gran ausente es la tercera categoría propuesta por Glänzel (2003), asociada a una bibliometría orientada a la investigación básica o de fuerte componente metodológico. El hecho de que no existan publicaciones sobre este último aspecto puede deberse en gran medida a un menor desarrollo del campo de las metrías en América Latina y el Caribe y, por ende, una insuficiente masa crítica de investigadores que aborde este perfil. 
Figura 4. Perfil temático y naturaleza de las investigaciones (umbral de representación: palabras clave asociadas con una frecuencia $\geq$ a 2).

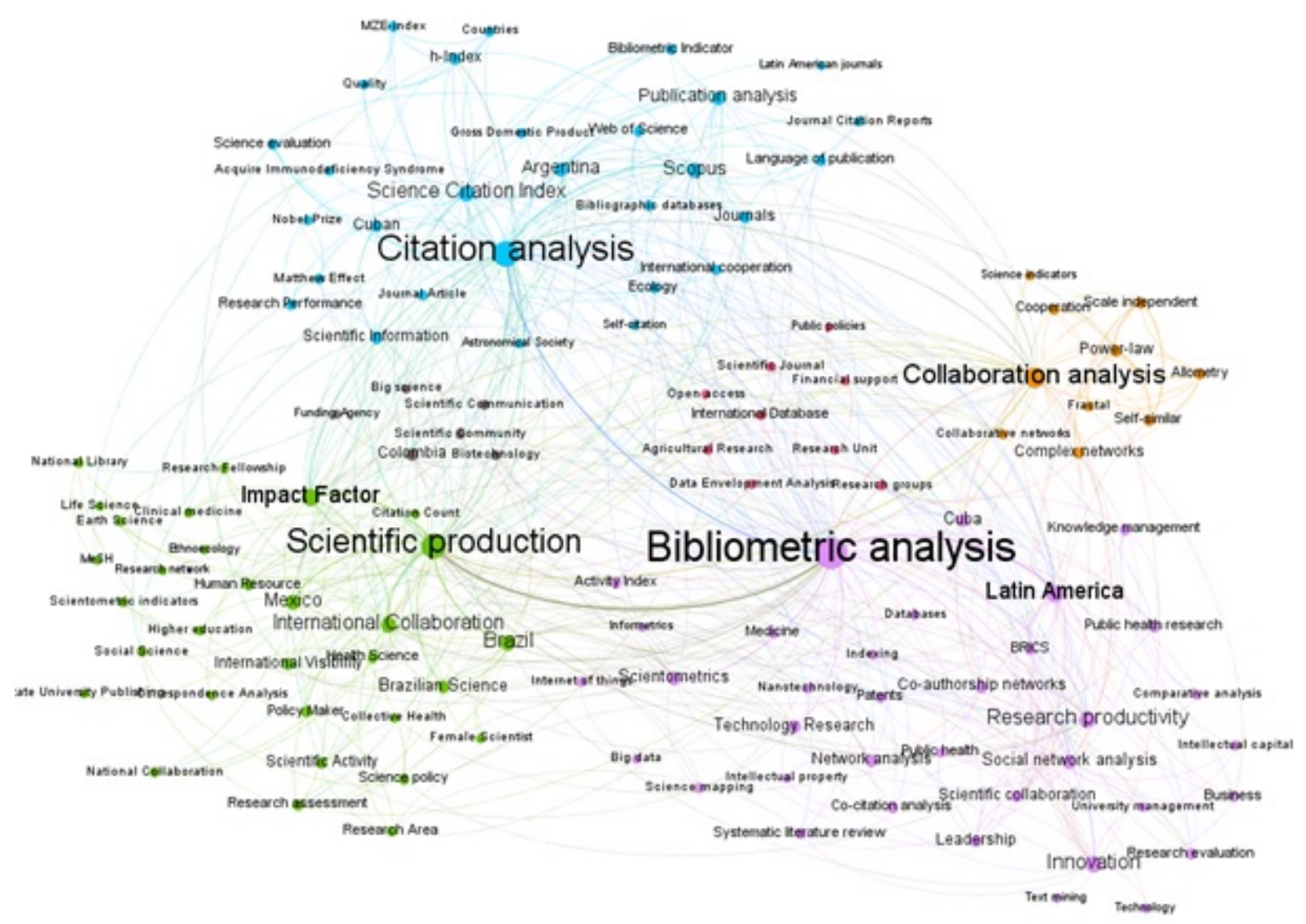

Fuente: elaboración propia.

Del análisis de cocitación de las fuentes incluidas en las referencias bibliográficas de la producción (Figura 5), los resultados arrojan que, por un lado, Scientometrics es la revista más influyente en la estructura que constituye la base intelectual de las contribuciones. Luego, aparecen solo tres revistas que coinciden con las más elegidas para publicar en Brasil, México, Colombia y Argentina según estudios previos (ver Tabla 1): Ciência da informação, Interciencia y REDC. Por otro lado, aparecen algunas revistas que conforman el núcleo de publicaciones de la literatura internacional sobre EMI: Journal of informetrics, JASIS\& T, Journal of information science, ARIST, Research evaluation, Research policy, Journal of documentation. Y finalmente, se observa la presencia de otros títulos que no están dentro de las publicaciones más influyentes del campo, por ejemplo, el grupo de títulos relacionado con la gestión de la ciencia, que aparecen cocitadas más fuertemente con Research policy; o el grupo de revistas relacionadas con las investigaciones de tecnología e innovación, que estarían vinculadas con algunos de los clusters observados en la visualización de las temáticas de las contribuciones. 
Figura 5. Mapa de cocitación de revistas de la producción de países de América Latina y el Caribe en la revista Scientometrics (1978-2020).

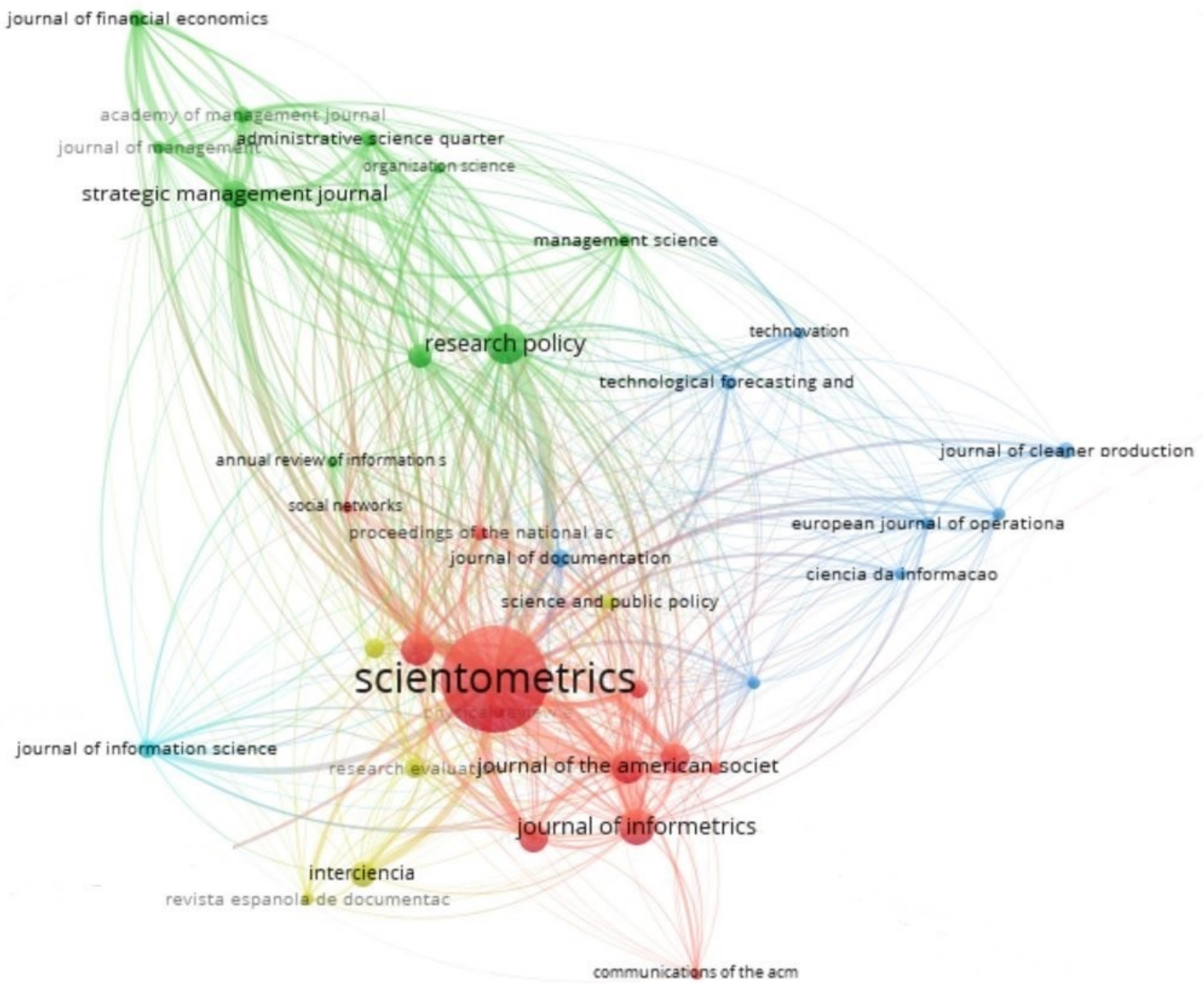

Fuente: elaboración propia.

\section{CONCLUSIONES Y DISCUSIÓN}

El número de contribuciones de autores latinoamericanos a la revista Scientometrics es escaso, especialmente teniendo en cuenta el volumen que reportan estudios previos para algunos países como Brasil, México, Colombia y Argentina (Miguel y Dimitri, 2013; Restrepo Arango y Urbizagástegui Alvarado, 2016; Urbizagástegui Alvarado, 2016; Urbizagástegui Alvarado y Restrepo Arango, 2013). Sin embargo, la presencia de autores de 11 países de la región con al menos una contribución en la revista pone de relieve el interés de la comunidad latinoamericana dedicada a los EMI en dialogar con la comunidad internacional especializada en este campo de estudios.

Si bien el mayor incremento de contribuciones se da a comienzos de la década de 2000, no todos los países tienen presencia en todo el período, sino que se observa un comportamiento de incorporación paulatina y fluctuante. En este sentido, el lapso de una década o más en la reaparición de un país en la revista podría dar cuenta de contribuciones esporádicas de algún investigador o equipo en algún momento de sus carreras académicas; mientras que la permanencia sostenida de un país podría revelar la existencia de equipos de investigación que se han ido consolidando en el campo de los EMI, y que han logrado hacer contribuciones tanto en la esfera nacional/regional como en la arena internacional.

En cuanto al perfil temático de la producción se observa, en general, un énfasis en los análisis bibliométricos con base en la aplicación de múltiples indicadores orientados a mapear dominios temáticos, geográficos e 
institucionales con el objetivo, en muchos casos, de observar aspectos relacionados con la innovación, las redes de colaboración, la productividad científica o el impacto de la producción publicada, entre otros. Esto se hace evidente al visualizar los clusters delimitados en el grafo de la red social mostrada, aunque esta representación debe ser interpretada a la luz de las limitaciones existentes en la precisión con que los autores etiquetan sus contribuciones que, como se señaló previamente, abundan en múltiples variantes de términos y expresiones referidas a métodos, técnicas e indicadores o, por el contrario, en síntesis extremas de los tópicos abordados. Aún así, resulta claro que los mayores intereses se centran en el estudio de las producciones indizadas en la corriente principal, aspecto que debe relacionarse con las políticas científicas implementadas por muchos países de la región que, desde una percepción periférica, intentan direccionar sus publicaciones hacia el espacio mainstream. Esto explica, en parte, un importante número de trabajos orientados al análisis de la visibilidad de las producciones por medio de la citación y el impacto, o el estudio de la colaboración científica en términos de la capacidad de apertura y asociación de las comunidades de investigadores en el marco de los crecientes procesos de internacionalización de la ciencia. El hecho de que estos artículos hayan sido publicados en una revista especializada de prestigio internacional como Scientometrics demuestra que las temáticas abordadas en ellos son homologables a los intereses y cobertura de esta publicación desde el punto de vista de las tendencias existentes en el campo de las metrías. No obstante, también se ha podido comprobar que la presencia de contribuciones de autores regionales se realiza en base a investigaciones sustentadas en una "bibliometría aplicada", orientada casi exclusivamente al diagnóstico y evaluación de dominios científicos y consistente con el perfil observado en estudios previos (Grácio y Oliveira, 2012; Miguel y Dimitri, 2013).

La ausencia de trabajos que incursionen en aspectos básicos a partir de aportes de índole metodológica hace suponer que la comunidad de investigadores especializados en los EMI es incipiente o no ha alcanzado la maduración suficiente. Sobre este último aspecto, es importante mencionar también que el desarrollo de líneas de investigación en este ámbito tiene una directa relación con la dimensión de los sistemas científicos nacionales de los países de ALyC, cuestión que queda patentizada con la procedencia geográfica de los autores más productivos.

En lo que respecta a la base intelectual de la producción, se concluye que los investigadores latinoamericanos que publican en Scientometrics utilizan como fuentes varias revistas internacionales que conforman el núcleo del campo de los EMI. Por otra parte, salvo el caso de Scientometrics, y otras pocas revistas como Ciência da informação, REDC e Interciencia, no hay mayor coincidencia entre las elegidas para publicar -según los estudios previos encontrados-, y las fuentes que constituyen la base intelectual de la producción; al menos, cuando publican en Scientometrics. Debería explorarse en otros estudios si estas fuentes también conforman la estructura intelectual cuando los autores publican en revistas nacionales o regionales. Finalmente agregar, que hacen falta más estudios para un conocimiento más completo de la producción científica en EMI de los países de la región, lo que podría dar mayor visibilidad a la comunidad latinoamericana que desarrolla investigación en este campo de estudios.

\section{REFERENCIAS}

Chen, Y., Börner, K. y Fang, S. (2013). Evolving collaboration networks in Scientometrics in 1978-2010: a micromacro analysis. Scientometrics, 95, 1051-1070. https://doi.org/10.1007/s11192-012-0895-2

Glänzel, W. (2003). Bibliometrics as a research field: a course on theory and application of Bibliometric indicators. Bélgica. Recuperado de http://citeseerx.ist.psu.edu/viewdoc/download?doi=10.1.1.97.5311\&rep=rep1\&type=pdf

Grácio, M. C. C. y Oliveira, E. F. T de. (2012). A inserção e o impacto internacional da pesquisa brasileira em 'estudos métricos': uma análise na base Scopus. Tendências da pesquisa brasileira em ciência da informação, 5(1), 1-19. Recuperado de http://hdl.handle.net/11449/114791

Martín-Martín, A., Orduña-Malea, E., Ayllón, J. M. y Delgado López-Cózar, E. (2016). The counting house: measuring those who count. Presence of bibliometrics, scientometrics, informetrics, webometrics and altmetrics 
in the Google Scholar citations, ResearcherID, ResearchGate, Mendeley \& Twitter. EC3 working papers, 21. Recuperado de https://arxiv.org/ftp/arxiv/papers/1602/1602.02412.pdf

Miguel, S. E. y Dimitri, P. (2013). La investigación en bibliometría en la Argentina: quiénes son y qué producen los autores argentinos que realizan estudios bibliométricos. Información, cultura y sociedad, 29, 117-138. https://d oi.org/10.34096/ics.i29.677

Patra, S. K., Bhattacharya, P. y Verma, N. (2006). Bibliometric study of literature on bibliometrics. Bulletin of information technology, 26(1), 27-32. https://doi.org/10.14429/djlit.26.1.3672

Restrepo Arango, C. y Urbizagástegui Alvarado, R. (2016). Acercamiento a los estudios Biliométricos, Cienciométricos e Informétricos en México. Informação \& sociedade: estudos, 26(1), 51-71. Recuperado de htt ps://periodicos.ufpb.br/ojs/index.php/ies/article/view/22799

Schoepflin, U. y Glänzel, W. (2001). Two decades of Scientometrics: an interdisciplinary field represented by its leading journal. Scientometrics, 50, 301-312. https://doi.org/10.1023/a:1010577824449

Urbizagástegui Alvarado, R. (2016). La bibliometría, informetría, cienciometría y otras “metrías" en el Brasil. Encontros bibli: revista eletrônica de biblioteconomia e ciência da informação, 21(47), 51-66. http://dx.doi.org/10.5007/1 518-2924.2016v21n47p51

Urbizagástegui Alvarado, R. y Restrepo Arango, C. (2013). El desarrollo de la bibliometria $y$ los indicadores en ciencia $y$ tecnologia en Colombia. En IX Congreso Iberoamericano de Indicadores de Ciencia y Tecnología, Bogotá, Colombia. Recuperado de https://www.academia.edu/10183615/EL_DESARROLLO_DE_LA_BIBLIOMETR\%C3\%8DA_Y_LO S_INDICADORES_EN_CIENCIA_Y_TECNOLOG\%C3\%8DA_EN_COLOMBIA

Urbizagástegui Alvarado, R. y Restrepo Arango, C. (2017). Crecimiento de la literatura sobre bibliometria, informetria y cienciometria en el Brasil. Revista ibero-americana de ciência da informação, 10(1), 6-31. Recuperado de https ://periodicos.unb.br/index.php/RICI/article/download/2469/2198/

Vinkler, P. (2019). Core journals and elite subsets in scientometrics. Scientometrics, 121, 241-259. https://doi.org/1 $0.1007 / \mathrm{s} 11192-019-03199-5$ 